\title{
Low Fin Tubes for Moisture-separator-reheater for Nuclear Power Plant*
}

\section{New Ti-stabilized Ferritic Stainless Steels for MSR Low Fin Tubes}

Use of ferritic stainless steels as low fin tube in the moisture-sepa-

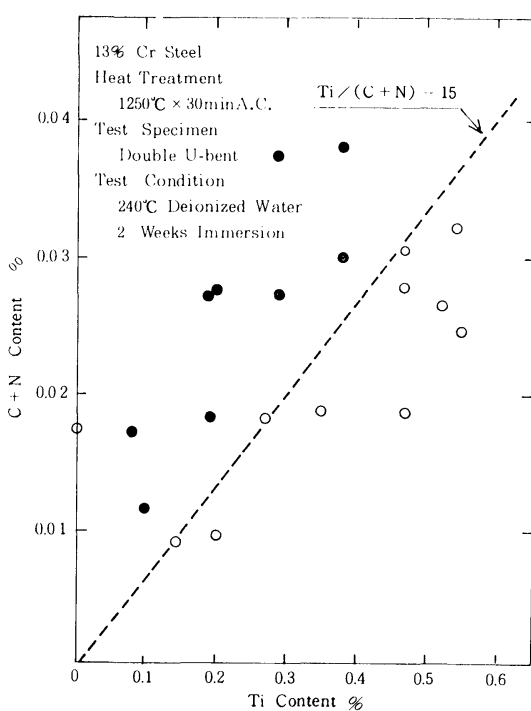

Fig. 1.

Effect of $\mathrm{Ti}$ and $(\mathrm{C}+\mathrm{N})$ content on $\mathrm{SCC}$ susceptibility of $13 \% \mathrm{Cr}$ steel in high temperature deionized water.

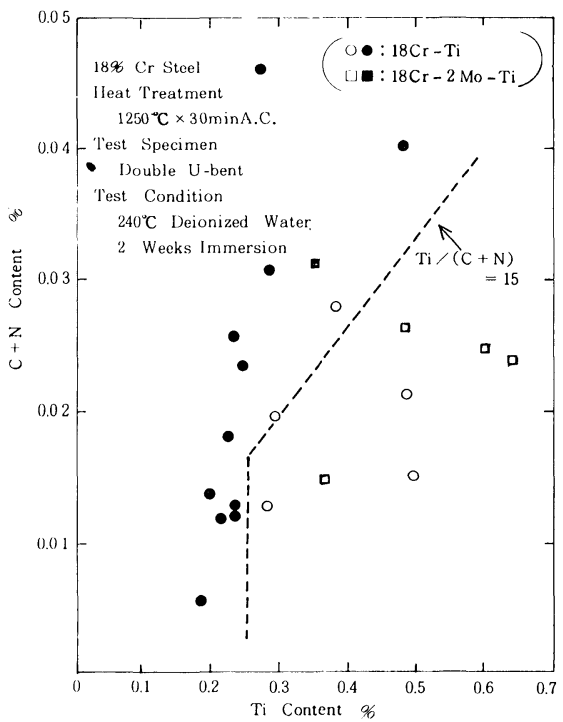

Fig. 2.

Effect of $\mathrm{Ti}$ and $(\mathrm{C}+\mathrm{N})$ content on $\mathrm{SCC}$ susceptibility of $18 \% \mathrm{Cr}$ steel in high temperature deionized water. rator-reheater (MSR) for nuclear power plant is on the increase for their superior corrosion resistance. However, they are known to become susceptible to intergranular corrosion, even the intergranular stress corrosion cracking (IGSGG) like the austenitic stainless steels, when sensitized and exposed to high temperature water containing dissolved oxygen, namely the service situation of the welding heat affected zones of the MSR heatexchanger tubes. At Kobe Steel, Ltd., it was found that the IGSCG could be prevented by adding $\mathrm{Ti}$ as a stabilizer for $\mathrm{C}$ and $\mathrm{N}$ and controlling the ratio of $\mathrm{Ti}$ to $(\mathrm{C}+$ $\mathrm{N})$.
The result of SCG test using double U-bent specimen is shown in Figs. 1 and 2.

These figures indicate that both the $13 \% \mathrm{Cr}$ and the $18 \% \mathrm{Cr}$ stainless steels become nonsusceptible to IGSGG if $\mathrm{Ti} /(\mathrm{C}+\mathrm{N})$ is at least 15 with $\mathrm{Ti}$ over $0.25 \%$

\section{Quality Level}

The MSR low fin tubes made from those new Ti-stabilized ferritic stainless steels are now available from Kobe Steel, Ltd. Chemical composition and quality level of products of ASTM A268 TP409 Mod. and TPXM-8 Mod. are shown in Tables 1 and 2.

Table 1. Chemical compositions of products.

\begin{tabular}{|c|c|c|c|c|c|c|c|c|c|c|c|c|}
\hline \multirow{2}{*}{ Grade } & \multirow{2}{*}{ Items } & \multicolumn{10}{|c|}{ Chemical Compositions $\%$} & \multirow{2}{*}{$\frac{\mathrm{Ti}}{\mathrm{C}+\mathrm{N}}$} \\
\hline & & $\mathrm{C}$ & $\mathrm{Si}$ & $\mathrm{Mn}$ & $\mathrm{P}$ & $\mathrm{S}$ & $\mathrm{Ni}$ & $\mathrm{Cr}$ & $\mathrm{N}$ & $\mathrm{Ti}$ & $\mathrm{Al}$ & \\
\hline \multirow{2}{*}{$\begin{array}{l}\text { 'TP409 } \\
\text { Mod. }\end{array}$} & $\begin{array}{l}\text { ASTM A268 } \\
\text { Standard }\end{array}$ & $\begin{array}{l}\text { Max } \\
0.08\end{array}$ & $\begin{array}{l}\operatorname{Max} \\
1.00\end{array}$ & $\begin{array}{c}\operatorname{Max} \\
1.0\end{array}$ & $\begin{array}{c}\text { Max } \\
0.045\end{array}$ & $\begin{array}{c}\text { Max } \\
0.045\end{array}$ & $\begin{array}{l}\operatorname{Max} \\
0.50\end{array}$ & $\begin{array}{l}10.50 \\
1.75\end{array}$ & & $\begin{array}{l}6 \times \underset{\mathrm{C}}{ } \\
0.75\end{array}$ & & \\
\hline & $\begin{array}{l}\text { Seamless } \\
\text { Tube }\end{array}$ & 0.010 & 0.42 & 0.55 & 0.024 & 0.010 & 0.11 & 12.31 & 0.0056 & 0.36 & 0.03 & 23.1 \\
\hline \multirow{2}{*}{$\begin{array}{c}\text { TPXM-8 } \\
\text { Mod. }\end{array}$} & $\begin{array}{l}\text { ASTM A268 } \\
\text { Standard }\end{array}$ & $\begin{array}{l}\operatorname{Max} \\
0.07\end{array}$ & $\begin{array}{l}\operatorname{Max} \\
1.00\end{array}$ & $\begin{array}{l}\text { Max } \\
1.00\end{array}$ & $\begin{array}{c}\text { Max } \\
0.040\end{array}$ & $\begin{array}{c}\text { Max } \\
0.030\end{array}$ & $\begin{array}{l}\operatorname{Max} \\
0.50\end{array}$ & $\begin{array}{l}17.00 \\
1.000\end{array}$ & & $\begin{array}{r}12 \times C \\
1.10\end{array}$ & $\begin{array}{l}\operatorname{Max} \\
0.15\end{array}$ & \\
\hline & $\begin{array}{l}\text { Seamless } \\
\text { Tube }\end{array}$ & 0.004 & 0.43 & 0.66 & 0.027 & 0.005 & 0.26 & 17.66 & 0.0086 & 0.28 & 0.03 & 22.2 \\
\hline
\end{tabular}

Table 2. Representative quality level of products.

\begin{tabular}{|c|c|c|c|c|}
\hline & \multirow[b]{2}{*}{ Items } & \multirow[b]{2}{*}{ Specification } & TP409 Mod. & TPXM-8 Mod. \\
\hline & & & $\begin{array}{l}\text { Seamless Tube } \\
(22.23 \mathrm{OD} \times 1.24 \mathrm{t})\end{array}$ & $\begin{array}{l}\text { Seamless Tube } \\
(19.09 \text { OD } \times 1.47 t)\end{array}$ \\
\hline \multirow[t]{4}{*}{ NDI } & UT(Before Finning) & \multirow{4}{*}{-} & Good & Good \\
\hline & ET (Before Finning) & & Good & Good \\
\hline & ET (After Finning) & & Good & Good \\
\hline & ALT(After Finning) & & Good & Good \\
\hline \multirow{2}{*}{$\begin{array}{l}\text { Dimensions } \\
\text { of Unfinned } \\
\text { Portion }\end{array}$} & $\begin{array}{l}\text { Outside Diameter } \\
\text { (DT) }\end{array}$ & \pm 0.1 & $22.15 \sim 22.31$ & $18.95 \sim 19.11$ \\
\hline & $\begin{array}{l}\text { Wall Thickness } \\
(\text { WT })\end{array}$ & $\begin{array}{l} \pm 10 \% \text { or } \pm 0.2 \\
\text { Whichever is greater }\end{array}$ & $\begin{array}{l}(1.83 \pm 0.2) \\
1.72 \sim 1.96\end{array}$ & $\begin{array}{l}(2.11 \pm 1096) \\
1.99 \sim 2.24\end{array}$ \\
\hline \multirow{4}{*}{$\begin{array}{l}\text { Dimensions } \\
\text { of Finned } \\
\text { Portion }\end{array}$} & $\begin{array}{l}\text { Outside Diameter } \\
\text { (DF) }\end{array}$ & $\begin{array}{l}+0.1 \\
-0.3 \\
\end{array}$ & $22.02 \sim 22.20$ & $18.83 \sim 19.00$ \\
\hline & $\begin{array}{l}\text { Wall Thickness } \\
\left(W_{F}\right)\end{array}$ & Min. Wall & $\begin{array}{l}(\operatorname{Min} 1.10) \\
1.26 \sim 1.35\end{array}$ & $\begin{array}{l}(\operatorname{Min} 1.25) \\
1.52 \sim 1.61\end{array}$ \\
\hline & Fin Height $\left(\mathrm{H}_{F}\right)$ & - & $1.32 \sim 1.42$ & $1.40 \sim 1.48$ \\
\hline & Fins per lnch & $26 \pm 1$ & $26.1 \sim 26.3$ & $26.0 \sim 26.2$ \\
\hline \multirow{6}{*}{$\begin{array}{l}\text { Mechanical } \\
\text { Properties } \\
\left(\begin{array}{l}\text { Unfinned } \\
\text { Portion }\end{array}\right)\end{array}$} & $\mathrm{YS}$ at RT & $\geqq 21.1$ & $23 \sim 26$ & $28 \sim 29$ \\
\hline & $\mathrm{TS}$ at RT & $\geqq 42.2$ & $43 \sim 45$ & $45 \sim 46$ \\
\hline & El at RT & $\geqq 20$ & $47 \sim 50$ & $45 \sim 46$ \\
\hline & $\mathrm{YS}$ at $300^{\circ} \mathrm{C}$ & - & $18 \sim 20$ & $19 \sim 20$ \\
\hline & TS at $300^{\circ} \mathrm{C}$ & - & $36 \sim 37$ & $38 \sim 39$ \\
\hline & $\begin{array}{l}\text { Hardness } \\
(\mathrm{H} R \mathrm{~B})\end{array}$ & $\begin{array}{l}\text { TP409: } \leqq 95 \\
\text { TPXM }-8: \leqq 90\end{array}$ & $63 \sim 67$ & $73 \sim 75$ \\
\hline
\end{tabular}

* For further information, write to Steel Tubes \& Pipes Technical Department, Kobe Steel, Ltd., 1-8-2, Marunouchi, Chiyoda-ku, Tokyo 100. (C) 1986 ISIJ 